Article (refereed) - postprint

Rickhaus, Peter; Wallbank, John; Slizovskiy, Sergey; Pisoni, Riccardo; Overweg, Hiske; Lee, Yongjin; Eich, Marius; Liu, Ming-Hao; Watanabe, Kenji; Taniguchi, Takashi; Ihn, Thomas; Ensslin, Klaus. 2018. Transport through a network of topological channels in twisted bilayer graphene. Nano Letters, 18 (11). 6725-6730. https://doi.org/10.1021/acs.nanolett.8b02387

Copyright (c) 2018 American Chemical Society

This version available http://nora.nerc.ac.uk/id/eprint/521661/

NERC has developed NORA to enable users to access research outputs wholly or partially funded by NERC. Copyright and other rights for material on this site are retained by the rights owners. Users should read the terms and conditions of use of this material at http://nora.nerc.ac.uk/policies.html\#access

This document is the author's final manuscript version of the journal article following the peer review process. Some differences between this and the publisher's version may remain. You are advised to consult the publisher's version if you wish to cite from this article.

https://pubs.acs.org/journal/nalefd 


\title{
Transport Through a Network of Topological Channels in Twisted Bilayer Graphene
}

\author{
Peter Rickhaus, ${ }^{*}, \dagger$ John Wallbank, ${ }^{\ddagger}$ Sergey Slizovskiy, ${ }^{\top}$ Riccardo Pisoni, ${ }^{\dagger}$ Hiske \\ Overweg, ${ }^{\dagger}$ Yongjin Lee, ${ }^{\dagger}$ Marius Eich, ${ }^{\dagger}$ Ming-Hao Liu, ${ }^{\S}$ Kenji Watanabe,॥ \\ Takashi Taniguchi, ${ }^{\text {Thomas Ihn }}{ }^{\dagger}$ and Klaus Ensslin ${ }^{\dagger}$ \\ †Department of Physics, ETH Zürich, Otto-Stern-Weg 1, 8093 Zürich, Switzerland \\ $\ddagger$ Centre for Ecology and Hydrology, Maclean Building, Benson Lane, Crowmarsh Gifford, \\ Wallingford, Oxfordshire, OX10 8BB, UK \\ 9NRC "Kurchatov Institute" PNPI, Gatchina, 188300, Russia \\ $\S$ Department of Physics, National Cheng Kung University, Tainan 70101, Taiwan \\ || National Institute for Material Science, 1-1 Namiki, Tsukuba 305-0044, Japan \\ E-mail: peterri@phys.ethz.ch
}

\begin{abstract}
We explore a network of electronic quantum valley Hall (QVH) states in the moiré crystal of minimally twisted bilayer graphene. In our transport measurements we observe Fabry-Pérot and Aharanov-Bohm oscillations which are robust in magnetic fields ranging from 0 to $8 T$, in strong contrast to more conventional $2 \mathrm{D}$ systems where trajectories in the bulk are bent by the Lorentz force. This persistence in magnetic field and the linear spacing in density indicate that charge carriers in the bulk flow in topologically protected, one dimensional channels. With this work we demonstrate coherent electronic transport in a lattice of topologically protected states.
\end{abstract}


Keywords: Twisted bilayer graphene; Topological network; Fabry-Pérot, Valleytronics; Moiré superlattice; Quantum valley Hall effect
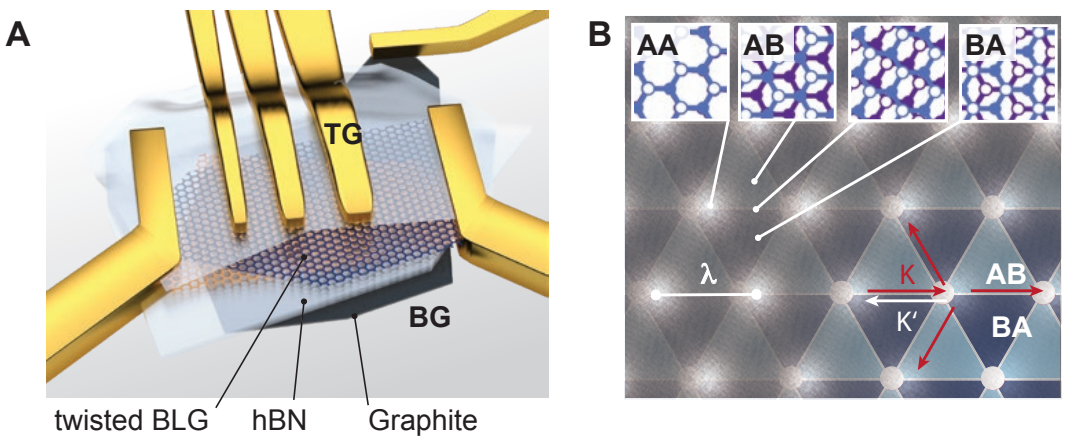

Figure 1: Schematics of the measured device. A, twisted bilayer graphene (tBLG) is encapsulated in boron-nitride (hBN). Using the BG, the global density $n_{\text {out }}$ can be changed, while the TGs allow to change $n_{\text {in }}$ and $D$. B , Two hexagonal lattices are twisted by $\theta=0.42$, giving rise to a moiré periodicity of $\lambda=33 \mathrm{~nm}$. By depleting $\mathrm{AB}$ and $\mathrm{BA}$ regions, helical currents connecting the AA points, arise.

Topological channels ${ }^{1-4}$ hold promises for quantum computation with reduced decoherence. In order to create topological states in bilayer graphene (BLG), a large displacement field $D$ has to be applied between the two layers. By this a band-gap opens around the charge neutrality point. The geometric boundaries at which helical states then emerge are given by stacking faults, ${ }^{5-12}$ a smooth transition between $\mathrm{AB}$ and $\mathrm{BA}$ stacking regions ${ }^{13}$ or the local inversion of $D \cdot{ }^{14-16}$ In a moiré crystal of twisted bilayer graphene (tBLG), alternating regions of $\mathrm{AB}$ and $\mathrm{BA}$ stacking naturally exist and they form a superlattice. ${ }^{17}$ The $\mathrm{AB}$ and $\mathrm{BA}$ regions can be depleted by applying large $D$ and the emerging states form a network, ${ }^{17,18}$ as recently shown by STM measurements. ${ }^{19}$ This network forms due to different valley Chern numbers in the $\mathrm{AB}$ and $\mathrm{BA}$ stacking regimes. The condition for its formation is that the twist angle is sufficiently small, such that the size of the $\mathrm{AB} / \mathrm{BA}$ regions is large. First theories suggested that twist angles $\theta<0.3$ are required,${ }^{17}$ however, elastic deformations stabilize and enlarge the $\mathrm{AB} / \mathrm{BA}$ regions and therefore relax this condition. ${ }^{18}$ This is in contrast to the emergence of superconductivity ${ }^{20}$ which requires a magical twist angle around 1. Compared to other helical systems, the topological currents flow predominately in the bulk of the sample. This brings the advantage that the system is less sensitive to 
impurities that originate from processing the sample edge (e.g. in InAs/GaSb systems ${ }^{21}$ ).

We probe the topological network using a Fabry-Pérot cavity, formed by a backgate (BG) and a local topgate (TG), and measure charge carrier transmission in a linear conductance experiment. Interfaces between bulk and cavity are semi-transparent, leading to standing waves. ${ }^{22-24}$ We observe magneto-conductance oscillations that are tuned by density $n$ (FabryPérot resonances) and magnetic field $B$ (Aharanov-Bohm resonances). The Fabry-Pérot resonances, at $n$ close to zero, are periodic in $n$ (rather than $\sqrt{n}$ ) demonstrating the 1D (rather than 2D) nature of the corresponding channels. Upon application of $B$, AharanovBohm oscillations arise with characteristic areas much smaller than the cavity size but also much larger than the moiré unit cell. We find that the characteristic orbits are in the cavity bulk, encompassing several unit cells. In other systems, Fabry-Pérot resonances are typically suppressed once the cyclotron diameter becomes comparable to relevant device dimensions. ${ }^{25,26}$ In our experiments they persist up to $B=8 T$ where the magnetic length $(9 \mathrm{~nm})$ is much smaller than any device dimension. The fact that oscillations nonetheless persist indicates that time reversal symmetry cannot be broken or that there is another protective symmetry at play. This hints at topological protection of corresponding 1D states. Our claims are substantiated by band structure calculations.

The measured device is schematically drawn in Fig. 1A (details in Fig. S1). tBLG is encapsulated in hBN and contacted with $\mathrm{Cr} / \mathrm{Au} \cdot{ }^{27}$ The bulk carrier density $n_{\text {out }}$ can be adjusted using a BG. ${ }^{28}$ Three TGs having lithographic lengths $L=200,300,400 \mathrm{~nm}$, allow to adjust cavity density $n_{\text {in }}$ and displacement field $D . n_{\text {in }}$, is tuned by the voltages on the topgate and on the graphite backgate, $V_{\mathrm{tg}}$ and $V_{\mathrm{bg}}$ respectively, according to the equation: $n_{\text {in }}=\left(C_{\mathrm{tg}} V_{\mathrm{tg}}+C_{\mathrm{bg}} V_{\mathrm{bg}}\right) / e$. The capacitances per unit area are determined from a parallel plate capacitor model, i.e. $C_{\mathrm{tg}}=\epsilon_{0} \epsilon_{\mathrm{hBN}} / d_{\mathrm{top}}$ and $C_{\mathrm{bg}}=\epsilon_{0} \epsilon_{\mathrm{hBN}} / d_{\mathrm{bottom}}$, where we use $\epsilon_{\mathrm{hBN}}=3.2$ and the $\mathrm{hBN}$ thicknesses $d_{\mathrm{top}}=27 \mathrm{~nm}, d_{\mathrm{bottom}}=45 \mathrm{~nm}$. To determine the displacement field, we use the simple approximation $D=\left(D_{\text {top }}-D_{\text {bottom })} / 2\right.$ and $D_{\text {top }}=\epsilon_{r} V_{\mathrm{TG}} / d_{\text {top }}$. The tBLG flake is etched to $W=4.6 \mu m$ giving cavities with $L \ll W$. Therefore, many parallel 
channels follow the same interference condition and standing waves in transport direction dominate the conductance.

The small twist angle is obtained by tearing a large graphene flake in the middle and picking up one half. The remaining part is twisted by $\theta=0.5$ and also picked up, following the procedure described in references. ${ }^{29,30}$ It is this careful fabrication that guarantees a well controlled and homogeneous moiré periodicity (a detailed description is given in the supplementary information Fig. S2).

Conductance measurements are performed using a standard low-frequency lock-in technique at $1.5 K$. From the Hofstadter butterfly pattern (Fig. S3) we extract a density $\left|n_{2}\right| \approx 0.8 \mathrm{e} 12 \mathrm{~cm}^{-2}$ at which the first band is completely filled, ${ }^{31}$ corresponding to $\theta=0.42$. In Fig. 1B, two hexagonal lattices, twisted by 0.42 are shown, exhibiting a large period moiré superlattice.

A

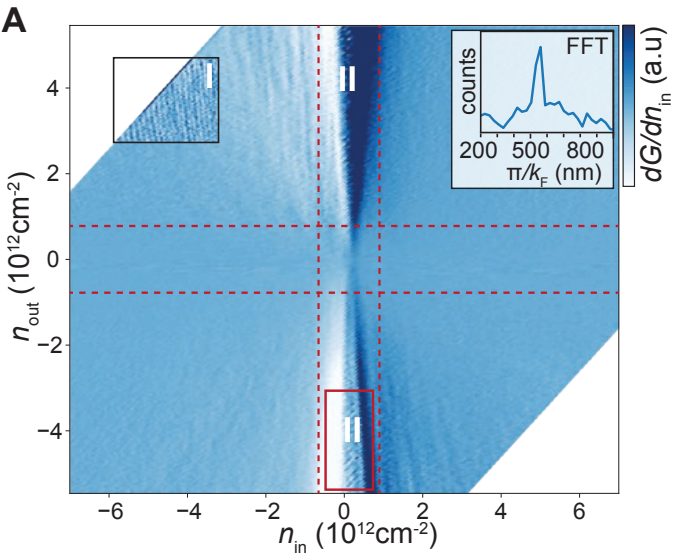

B

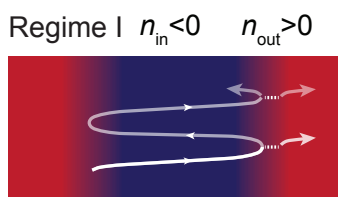

Regime II: $n_{\text {in }} \sim 0 \quad n_{\text {out }}>0$

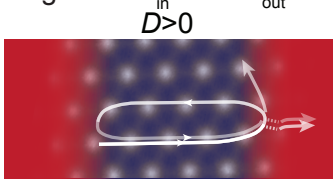

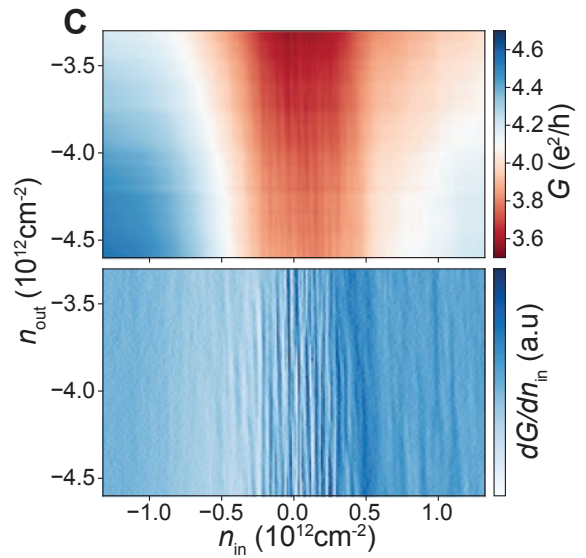

Figure 2: Fabry-Pérot (FP) oscillations measured at $1.5 K$. A, Differential conductance $d G / d n_{\text {in }}\left(n_{\text {in }}, n_{\text {out }}\right)$. Red dashed lines denote the density $n_{2}$. In regime I, FP oscillations appear for $n_{\text {in }} \ll 0$ and $n_{\text {out }} \gg 0$, as can be seen in the high-resolution scan in the marked window. The corresponding FFT (averaged over $n_{\text {out }}$ ) is shown in the inset. $\mathbf{B}$, FP resonances are due to standing waves in a cavity formed by the topgate. In regime II, the presence of topological channels is expected. C, A zoom into regime II (marked with a red solid square in $\mathbf{A}$ ) is shown (above: $G$, below: $d G / d n_{\text {in }}$ ).

In the measurement $d G / d n_{\text {in }}\left(n_{\text {in }}, n_{\text {out }}\right)$ (Fig. $\left.2 \mathrm{~A}\right),\left|n_{2}\right|$ is marked with red dashed lines. We first focus on the bipolar $n-p-n$ regime $\mathrm{I}$, where the densities $n_{\text {in }}, n_{\text {out }}$ are large but have opposite signs (a negative sign is used for charge carriers occurring at energies smaller 
zero). The displacement field can, but does not have to be large in this regime. For a semi-transparent interface, standing waves form as sketched in Fig. 2B, following the 2DFabry-Pérot interference condition $2 L=j \cdot 2 \pi / k_{\mathrm{F}}$ where $j=1,2, \ldots$ and $k_{\mathrm{F}} \approx \sqrt{n \pi}$. The observed pattern is very similar to measurements in mono- ${ }^{24,32,33}$ and bilayer graphene. ${ }^{34}$ The extracted cavity length $L=550 \mathrm{~nm}$ (see inset), is larger than the designed $L=400 \mathrm{~nm}$. This discrepancy is due to the smooth transition between cavity and bulk and is analyzed in detail in the supplementary material of reference. ${ }^{33}$ The observation of standard Fabry-Pérot oscillations in regime I shows that ballistic cavities with standing waves form.

We now focus on oscillations at small $n_{\text {in }}<n_{2}$ and large D (regime II, Fig. 2C). These resonances occur in a regime where we expect that the $\mathrm{AB} / \mathrm{BA}$ regions are depleted and the super-lattice symmetries affect the behavior in $B$.
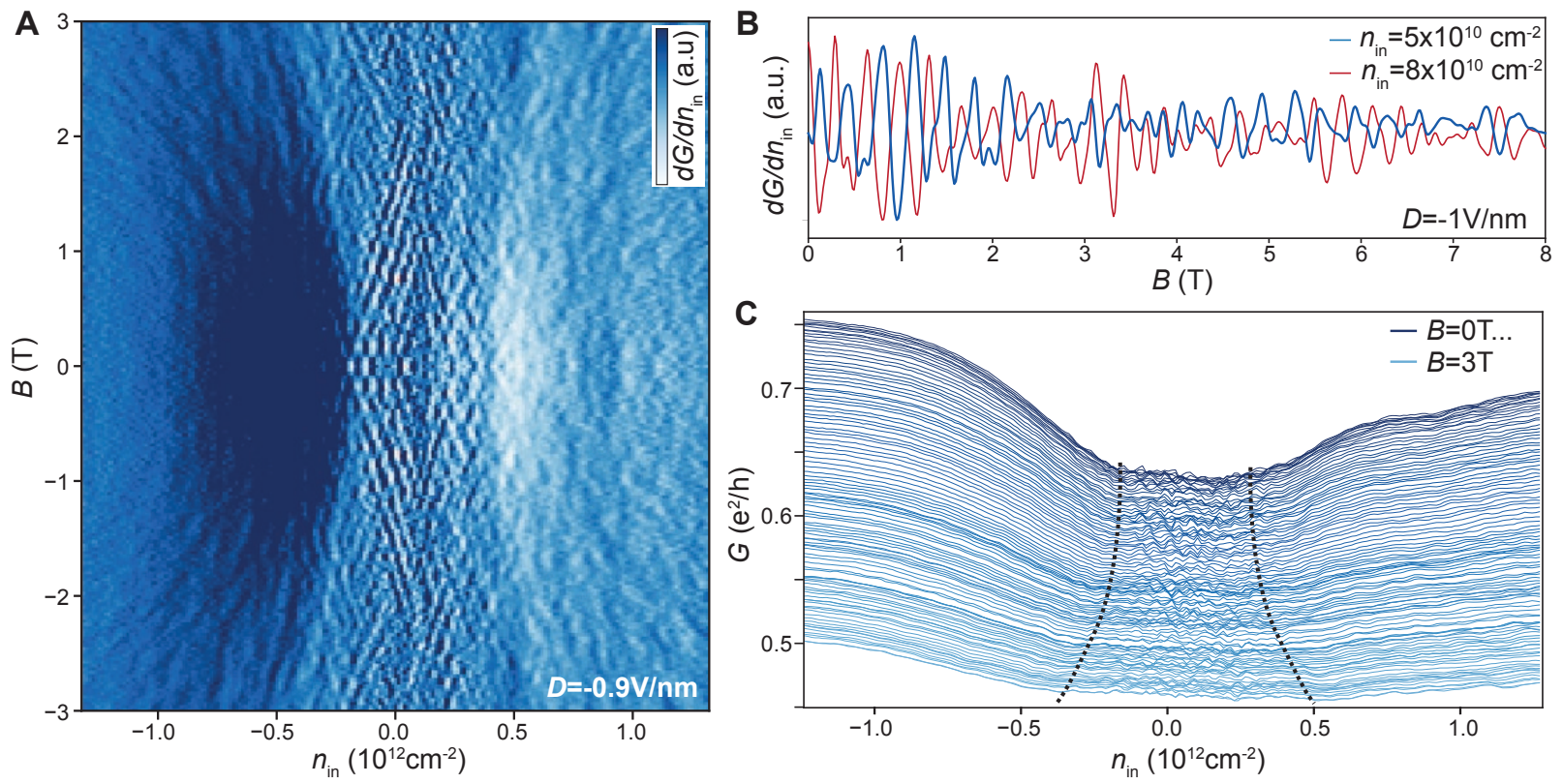

Figure 3: Magneto-conductance oscillations. A, A crossed resonance pattern emerges from oscillations at low $n_{\text {in }}$ and large $D$. B, Two lines $d G / d n_{\text {in }}(B)$ for fixed $n_{\text {in }}$ with an extended B-field range. Maxima and minima alternate up to $B=8 T$. C, $G\left(n_{\text {in }}\right)$ traces reveal that the background-conductance is nearly independent of $n_{\text {in }}$.

In a perpendicular magnetic field, trajectories of charge carriers, bouncing between two semi-transparent mirrors, bend due to the Lorentz force. Standard Fabry-Pérot oscillations 
require a cyclotron diameter $2 R_{c}:{ }^{25}$

$$
2 R_{\mathrm{c}}=2 \frac{\hbar k_{\mathrm{F}}}{e B}>L
$$

In our system, this condition holds true in regime I (see Fig. S4), where oscillations have vanished for $B>0.5 T$, but not in regime II. There, the corresponding magnetoconductance map (Fig. 3A) reveals a periodic pattern of crossed resonances, evolving continuously from 0 to $\pm 3 T$. The crossed pattern is formed by diagonal lines of opposite slope in the $n$ - $B$-plane. For the given density range, $2 R_{\mathrm{c}} \geq L$ for $B \lesssim 0.4 T$, the resonance pattern apparently neither disappears nor changes at $0.4 T$, but persists up to at least $8 T$ as seen in Fig. $3 \mathrm{~B}$ where we depict two traces $d G / d n_{\text {in }}(B)$ for slightly different values of $n_{\text {in }}$ (for clarity, a smoothened background has been removed). Up to a magnetic field of $8 T$ (and presumably beyond, $8 T$ was the maximum available field in our cryostat) the maxima and minima alternate periodically.

In other two-dimensional systems, resonances that depend on $B$ and $n$ at high magnetic fields (i.e. in the quantum Hall regime) were attributed to either single-electron charging of Landau levels in confined geometries or to Aharanov-Bohm interferences. ${ }^{35,36}$ These two effects are distinguished by the sign of slope in the $n-B$-plane. ${ }^{35}$ In our measurements, oscillations display both, positive and negative slopes simultaneously (Fig. 3A) and are therefore inconsistent with electron charging as a possible origin. For the case of Aharanov-Bohm oscillations however, a crossed pattern can be explained if the corresponding area is encircled both clock- and counterclockwise. In contrast to the above mentioned measurements, ${ }^{35,36}$ the resonances persist from the Quantum Hall regime down to low magnetic fields (Fig. 3A,B), and are thus not linked to the existence of Quantum Hall edge channels. This is a strong indication that the charge carriers already flow in one-dimensional channels for all magnetic field considered such that their trajectories remain unaffected by the magnetic field. Another, yet weaker, indication for one-dimensional transport is seen from the conductance 
traces (Fig. 3C) which are rather flat in the regime where the AB/BA regions are gapped (marked with dashed borders). This indicates that the number of conducting channels does not change with $n_{\text {in }}$ which is again consistent with a fixed number of one-dimensional channels.

More quantitative information can be obtained from the resonance periods in magnetic field and density. These are linked to the encircled area $A$ and the total length $L_{\text {tot }}$ of the coherent trajectories by the Bohr-Sommerfeld resonance condition:

$$
j=L_{\mathrm{tot}} \frac{k_{\mathrm{F}}}{2 \pi} \pm A \frac{B}{\phi_{0}}
$$

where $j$ is an integer and $\phi_{0}=h / e$. The spacing between two maxima is then given by $j-(j-1)=L_{\text {tot }} \frac{\Delta k_{\mathrm{F}}}{2 \pi} \pm A \frac{\Delta B}{\phi_{0}}$. From $\Delta B=0.37 T$ (extracted from Fig. 3B) we obtain $A=\phi_{0} / \Delta B=11200 \mathrm{~nm}^{2}$. This area is much larger than the area of a moiré unit cell, i.e. $\approx 950 \mathrm{~nm}^{2}$. On the other hand, the entire area of the top-gated cavity is $L \cdot W \approx 2 e 6 n m^{2}$ which is two orders of magnitude too large. Consequently, the interfering paths must be located in the cavity bulk.

By analyzing the spacing in density, $\Delta n_{\text {in }}$, we can extract information about the length $L_{\text {tot }}=2 \pi / \Delta k_{\mathrm{F}}$ of the interference path. Importantly, $k_{\mathrm{F}} \sim n_{\text {in }}\left(\right.$ not $\left.k_{\mathrm{F}} \sim \sqrt{n_{\mathrm{in}}}\right)$ since charge carriers flow in one-dimensional (not two-dimensional) channels. This leads to resonances following diagonal lines in the $n_{\mathrm{in}}-B$-plane $\left(k_{\mathrm{F}} \sim \sqrt{n_{\mathrm{in}}}\right.$ would lead to parabolic lines in the magnetoconductance map, which is not observed). To convert $n_{\text {in }}$, which is the (twodimensional) density in the twisted bilayer graphene flake tuned by the gate voltages, into a one-dimensional density $n_{1 \mathrm{D}}$ we divide by the number of channels per unit area, $N_{\text {ch }}=2 \sqrt{3} / \lambda$ (for details see supporting information, Eq. 4). To do so we use the moiré periodicity $\lambda=33 \mathrm{~nm}$ obtained from the Hofstadter butterfly, Fig. S3. For $\Delta n_{\text {in }}=4.7 e 10 \mathrm{~cm}^{-2}$ and using $L_{\text {tot }}=2 \pi / \Delta k_{\mathrm{F}}=8 \sqrt{3} /\left(\lambda \Delta n_{\text {in }}\right)$ we obtain $L_{\text {tot }} \approx 870 \mathrm{~nm}$.

The extracted area and circumference correspond to trajectories that encircle a long and 
narrow object. For a rectangle, it is straightforward to calculate the corresponding length $\tilde{L}=408$ and width $\tilde{w}=27 \mathrm{~nm}$. We note here that these values are close to the designed cavity length $L=400 \mathrm{~nm}$ and the height of the moiré unit cell $\lambda \sqrt{3} / 2=29 \mathrm{~nm}$ which also corresponds to the shortest distance between two topological channels. This suggests that one row of $\mathrm{AB} / \mathrm{BA}$ regions is encircled. However, also other trajectories are possible. In the topological network there are three valley-preserving scattering possibilities (red arrows in Fig. 1B) at every 'node' (AA stacking region). This allows for large and complex paths in the network. Especially, paths that do not require intervalley scattering (see discussion in Fig. S7B) are possible. However, closed trajectories consistent with the extracted area and length are long and narrow and if they do connect the two cavity interfaces then the cavity length $L$ is an important parameter. Since $A / L \approx \lambda \sqrt{3} / 2$ and $L_{\text {tot }}-2 L \approx 2 \lambda$ this is the only kind of trajectory that is consistent with our experimental results.
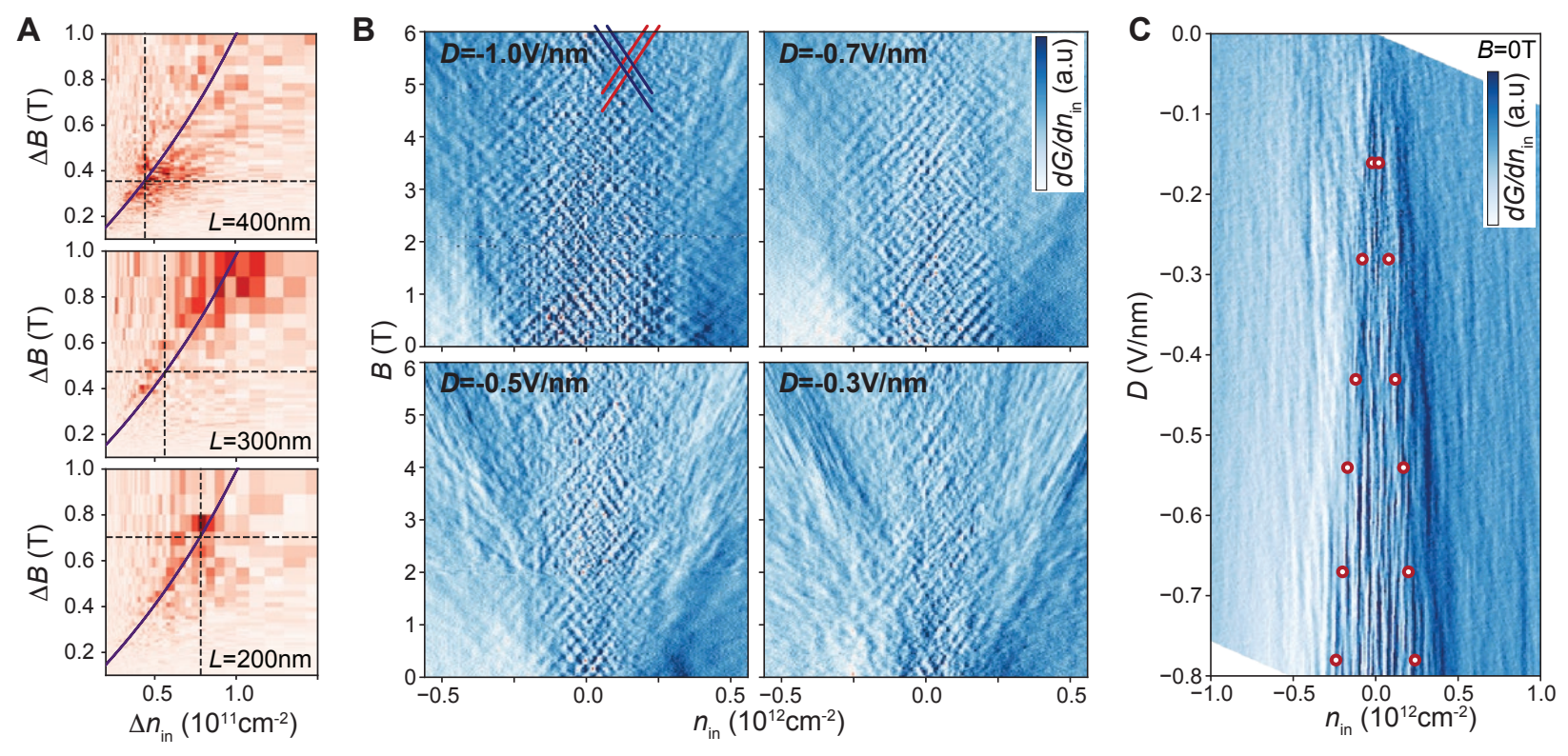

Figure 4: Dependence on $L$ and $D$. A, 2D FFT of magneto-conductance maps for $D=$ $-1 \mathrm{~V} / \mathrm{nm}$ and $L=200,300,400 \mathrm{~nm}$. The solid line shows the Bohr-Sommerfeld quantization for trajectories with varying length encircling one row of $\mathrm{AB} / \mathrm{BA}$ regions. The dashed lines depict the expected $\Delta B$ and $\Delta n$ for the designed $L$. B, $d G / d n_{\text {in }}$ for decreasing $D$ and $L=400 \mathrm{~nm}$. The $n_{\text {in }}$ range where the interferences are observed is shrinking. C, Interference pattern as a function of $D$ and $n_{\text {in }}$. Red dots mark the theoretically expected boundary for the resonances. 
By measuring the crossed resonance pattern with different topgates, it is possible to see how (and if) the resonance pattern depends on the designed cavity length $L$. In Fig. $4 \mathrm{~A}$ we show the results of a 2D fast Fourier transform (FFT) of magneto-conductance oscillations for cavities with $L=400,300,200 \mathrm{~nm}$. Apparently, the oscillations have a strong dependence on $L$ and become slower in both $\Delta B$ and $\Delta n_{\text {in }}$ for decreasing $L$, meaning that both the encircled area $A$ and circumference decrease in size. As a guide to the eye we depict the value of $\Delta B$ and $\Delta n_{\text {in }}$, that we would expect for a given $L$ by assuming that one row of $\mathrm{AB} / \mathrm{BA}$ regions is encircled, with dashed lines. The solid line is for arbitrary $L$ (details are given in the supplementary material). The measurements for $\Delta B$ and $\Delta n_{\text {in }}$ for different cavities appear to be consistent with straight parallel trajectories encircling one row of $\mathrm{AB} / \mathrm{BA}$ regions. Even though the states below the $300 \mathrm{~nm}$-sized topgate seem to resonate in an effectively shorter cavity, their trajectories also seem to encircle one row of $\mathrm{AB} / \mathrm{BA}$ regions as can be seen from the agreement with the solid line.

Finally we discuss the dependence on $D$. The measurements in Fig. 4B,C show that the resonance-pattern boundaries move closer when decreasing $D$. Such behavior is expected within the topological model. By lowering $D$, the induced gap size $\Delta$ (values in Fig. S9) in the $\mathrm{AB} / \mathrm{BA}$ regions shrinks and these regions start to become populated already at lower $n_{\text {in }}$. At the boundary of the resonance pattern, the Fermi-surface looses its one-dimensional character, as indicated by smearing of Fermi velocities projected onto the direction of 1D channel (supporting information Fig. S10B). This leads to dephasing and smearing of the interference pattern (see Fig. S10C). In Fig. 4C the densities, where the calculated Fermi velocity smears strongly, are marked with red dots, providing good agreement with the experimental data.

Conclusion We have fabricated tBLG with a twist angle of $\theta \approx 0.4$. We measured a crossed interference pattern which we explained by Aharanov-Bohm and Fabry-Pérot oscillations of trajectories that encircle an area clock- and anti-clockwise. The interference 
pattern persists from zero to large magnetic fields, which indicates that the charge carriers flow in one-dimensional channels. From the oscillation period in density and field we calculated area and circumference of the (dominant) resonant paths and found that their length is comparable to the cavity length and the width to the moiré periodicity. Similar loops are found for different gate lengths. The range (in density) within which the oscillations can be observed exhibits a dependence on displacement field that is consistent with the opening of a gap in $\mathrm{AB}$ and $\mathrm{BA}$ regions of the twisted bilayer graphene. Our observations are good indications that electrons form coherent paths within a network of topological channels that originates from the moiré superlattice.

Networks of helical channels offer several advantages for topologically protected quantum states: The two-dimensional nature of the network allows to perform complex valleytronic operations and, as demonstrated in this work, stabilizes coherent bulk transport phenomena such as Fabry-Pérot oscillations in magnetic field and against disorder. Furthermore, avoiding the physical edge leads to a better defined environment which improves topological protection. Our carbon based system is flexible, making it an important building block for scalable and protected valleytronic devices.

\section{Acknowledgments}

We thank Lucian Covaci, Beat Braem, Andreas Baumgartner and Lujun Wang for fruitful discussions and Peter Märki, Erwin Studer and FIRST staff for their technical support.

Funding: We acknowledge financial support from the European Graphene Flagship, the Swiss National Science Foundation via NCCR Quantum Science and Technology (QSIT) and ETH Zürich via the ETH fellowship program and the Taiwan Minister of Science and Technology (MOST) under Grant No. 107-2112-M-006 -004 -MY3. Growth of hexagonal boron nitride crystals was supported by the Elemental Strategy Initiative conducted by the MEXT, Japan and JSPS KAKENHI Grant Number JP15K21722. 
Authors contributions: PR fabricated the devices and performed the measurements. JW calculated the band structure. SS and M-HL helped to develop the theoretical understanding. SS and PR derived eq. 5,8 in SI. SS calculated gap size, Fermi-velocities and the parameter

b (eq. 9,10). RP, HO, YL and ME supported device fabrication and data analysis. KW and TT provided high-quality Boron-Nitride. KE and TI supervised the work.

Competing interests: Authors have no competing interests

Data and materials availability: All data is available in numerical form upon request.

\section{Supporting Information:}

Details on device design

Relative orientation of the moiré lattice and the TG/BG interface

Device fabrication and homogeneity of the moiré pattern

Hofstadter butterfly

Fabry-Pérot resonances of region II in $B$

Measurement of another sample

Calculation of Fabry-Pérot area and length

Model for one row of $\mathrm{AB} / \mathrm{BA}$ regions

Formation of semi-transparent mirrors at the device boundaries

Discussion of the band structure

Displacement field, bandgap and energy scales

Relation of $\Delta B$ with $\Delta n$

\section{References}

(1) Kane, C. L.; Mele, E. J. Quantum Spin Hall Effect in Graphene. Phys. Rev. Lett. 2005, 95, 226801. 
(2) König, M.; Wiedmann, S.; Brüne, C.; Roth, A.; Buhmann, H.; Molenkamp, L. W.; Qi, X.-L.; Zhang, S.-C. Quantum spin hall insulator state in HgTe quantum wells. Science 2007, 318, 766-70.

(3) Young, A. F.; Sanchez-Yamagishi, J. D.; Hunt, B.; Choi, S. H.; Watanabe, K.; Taniguchi, T.; Ashoori, R. C.; Jarillo-Herrero, P. Tunable symmetry breaking and helical edge transport in a graphene quantum spin Hall state. Nature 2014, 505, 528-532.

(4) Sau, J. D.; Lutchyn, R. M.; Tewari, S.; Das Sarma, S. Generic New Platform for Topological Quantum Computation Using Semiconductor Heterostructures. Phys. Rev. Lett. 2010, 104, 40502.

(5) Yao, W.; Yang, S. A.; Niu, Q. Edge States in Graphene: From Gapped Flat-Band to Gapless Chiral Modes. Phys. Rev. Lett. 2009, 102, 096801.

(6) Zhang, F.; Jung, J.; Fiete, G. A.; Niu, Q.; MacDonald, A. H. Spontaneous Quantum Hall States in Chirally Stacked Few-Layer Graphene Systems. Phys. Rev. Lett. 2011, $106,156801$.

(7) Zhang, F.; MacDonald, A. H.; Mele, E. J. Valley Chern Numbers and Boundary Modes in Gapped Bilayer Graphene. Proc. Natl. Acad. Sci. 2013, 110, 10546 LP - 10551.

(8) Hattendorf, S.; Georgi, A.; Liebmann, M.; Morgenstern, M. Networks of ABA and ABC stacked graphene on mica observed by scanning tunneling microscopy. Surf. Sci. 2013, $610,53-58$.

(9) Vaezi, A.; Liang, Y.; Ngai, D. H.; Yang, L.; Kim, E. A. Topological edge states at a tilt boundary in gated multilayer graphene. Phys. Rev. X 2013, 3, 1-9.

(10) Alden, J. S.; Tsen, A. W.; Huang, P. Y.; Hovden, R.; Brown, L.; Park, J.; Muller, D. A.; McEuen, P. L. Strain solitons and topological defects in bilayer graphene. Proc. Natl. Acad. Sci. U. S. A. 2013, 110, 11256-60. 
(11) Ju, L.; Shi, Z.; Nair, N.; Lv, Y.; Jin, C.; Velasco, J.; Ojeda-Aristizabal, C.; Bechtel, H. A.; Martin, M. C.; Zettl, A.; Analytis, J.; Wang, F. Topological valley transport at bilayer graphene domain walls. Nature 2015, 520, 650-655.

(12) Yin, L.-J.; Jiang, H.; Qiao, J.-B.; He, L. Direct imaging of topological edge states at a bilayer graphene domain wall. Nat. Commun. 2016, 7, 11760.

(13) Wright, A. R.; Hyart, T. Robust one-dimensional wires in lattice mismatched bilayer graphene. Appl. Phys. Lett. 2011, 98, 251902.

(14) Zarenia, M.; Pereira, J.; Peeters, F.; de Aquino Farias, G. Topological confinement in an antisymmetric potential in bilayer graphene in the presence of a magnetic field. Nanoscale Res. Lett. 2011, 6, 452.

(15) Li, J.; Wang, K.; McFaul, K. J.; Zern, Z.; Ren, Y.; Watanabe, K.; Taniguchi, T.; Qiao, Z.; Zhu, J. Gate-controlled topological conducting channels in bilayer graphene. Nat. Nanotechnol. 2016, 11, 1060.

(16) Lee, J.; Watanabe, K.; Taniguchi, T.; Lee, H.-J. Realisation of topological zero-energy mode in bilayer graphene in zero magnetic field. Sci. Rep. 2017, 7, 6466.

(17) San-Jose, P.; Prada, E. Helical networks in twisted bilayer graphene under interlayer bias. Phys. Rev. B 2013, 88 .

(18) Andjelkovic, M. and Covaci, L. and Peeters, F. M. DC conductivity of twisted bilayer graphene: Angle-dependent transport properties and effects of disorder. Phys. Rev. Mater. 2018, 2, 34004.

(19) Huang, S.; Kim, K.; Efimkin, D. K.; Lovorn, T.; Taniguchi, T.; Watanabe, K.; Macdonald, A. H.; Tutuc, E.; Leroy, B. J. Emergence of Topologically Protected Helical States in Minimally Twisted Bilayer Graphene. arXiv:1802.02999v1 2018, 
(20) Cao, Y.; Fatemi, V.; Fang, S.; Watanabe, K.; Taniguchi, T.; Kaxiras, E.; JarilloHerrero, P. Unconventional superconductivity in magic-angle graphene superlattices. Nature 2018, 556, 43.

(21) Mueller, S.; Mittag, C.; Tschirky, T.; Charpentier, C.; Wegscheider, W.; Ensslin, K.; Ihn, T. Edge transport in InAs and InAs/GaSb quantum wells. Phys. Rev. B 2017, 96,75406 .

(22) Liang, W.; Bockrath, M.; Bozovic, D.; Hafner, J. H.; Tinkham, M.; Park, H. Fabry Perot interference in a nanotube electron waveguide. Nature 2001, 411, 665.

(23) Ji, Y.; Chung, Y.; Sprinzak, D.; Heiblum, M.; Mahalu, D.; Shtrikman, H. An electronic Mach-Zehnder interferometer. Nature 2003, 422, 415.

(24) Young, A. F.; Kim, P. Quantum interference and Klein tunnelling in graphene heterojunctions. Nat. Phys. 2009, 5, 222.

(25) Rickhaus, P.; Makk, P.; Liu, M.-H.; Tóvári, E.; Weiss, M.; Maurand, R.; Richter, K.; Schönenberger, C. Snake trajectories in ultraclean graphene p-n junctions. Nat. Commun. 2015, 6, 6470 .

(26) Lee, M.; Wallbank, J. R.; Gallagher, P.; Watanabe, K.; Taniguchi, T.; Falko, V. I.; Goldhaber-Gordon, D. Ballistic miniband conduction in a graphene superlattice. Science (80-. ). 2016, 353, 1526-1529.

(27) Wang, L.; Meric, I.; Huang, P. Y.; Gao, Q.; Gao, Y.; Tran, H.; Taniguchi, T.; Watanabe, K.; Campos, L. M.; Muller, D. A.; Guo, J.; Kim, P.; Hone, J.; Shepard, K. L.; Dean, C. R. One-dimensional electrical contact to a two-dimensional material. Science 2013, 342, 614-7.

(28) Overweg, H.; Eggimann, H.; Chen, X.; Slizovskiy, S.; Eich, M.; Pisoni, R.; Lee, Y.; Rickhaus, P.; Watanabe, K.; Taniguchi, T.; Fal'ko, V.; Ihn, T.; Ensslin, K. Electro- 
statically Induced Quantum Point Contacts in Bilayer Graphene. Nano Lett. 2017, 18, $553-559$.

(29) Kim, K.; Yankowitz, M.; Fallahazad, B.; Kang, S.; Movva, H. C. P.; Huang, S.; Larentis, S.; Corbet, C. M.; Taniguchi, T.; Watanabe, K.; Banerjee, S. K.; LeRoy, B. J.; Tutuc, E. van der Waals Heterostructures with High Accuracy Rotational Alignment. Nano Lett. 2016, 16, 1989-1995.

(30) Cao, Y.; Luo, J.; Fatemi, V.; Fang, S.; Sanchez-Yamagishi, J.; Watanabe, K.; Taniguchi, T.; Kaxiras, E.; Jarillo-Herrero, P. Superlattice-Induced Insulating States and Valley-Protected Orbits in Twisted Bilayer Graphene. Phys. Rev. Lett. 2016, 117, 116804.

(31) Kim, K.; DaSilva, A.; Huang, S.; Fallahazad, B.; Larentis, S.; Taniguchi, T.; Watanabe, K.; Leroy, B. J.; Macdonald, A. H.; Tutuc, E.; Kim, P.; Novoselov, K. S. Tunable moiré bands and strong correlations in small-twist-angle bilayer graphene. Proc. Natl. Acad. Sci. 2017, 114, 3364-3369.

(32) Rickhaus, P.; Maurand, R.; Liu, M.-H.; Weiss, M.; Richter, K.; Schönenberger, C. Ballistic interferences in suspended graphene. Nat. Commun. 2013, 4, 3342.

(33) Handschin, C.; Makk, P.; Rickhaus, P.; Liu, M.-H.; Watanabe, K.; Taniguchi, T.; Richter, K.; Schoenenberger, C. Fabry-Pérot Resonances in a Graphene/hBN Moireuperlattice. Nano Lett. 2017, 17, 328-333.

(34) Varlet, A.; Liu, M.-H.; Krueckl, V.; Bischoff, D.; Simonet, P.; Watanabe, K.; Taniguchi, T.; Richter, K.; Ensslin, K.; Ihn, T. Fabry-Pérot Interference in Gapped Bilayer Graphene with Broken Anti-Klein Tunneling. Phys. Rev. Lett. 2014, 113, 116601.

(35) Zhang, Y.; McClure, D. T.; Levenson-Falk, E. M.; Marcus, C. M.; Pfeiffer, L. N.; West, K. W. Distinct signatures for Coulomb blockade and Aharonov-Bohm interference in electronic Fabry-Perot interferometers. Phys. Rev. B 2009, 79, 241304. 
(36) Overweg, H.; Rickhaus, P.; Eich, M.; Lee, Y.; Pisoni, R.; Watanabe, K.; Taniguchi, T.; Ihn, T.; Ensslin, K. Edge channel confinement in a bilayer graphene n-p-n quantum dot. New J. Phys. 2018, 20, 013013. 